common iliac artery). The para-aortic SLNs rate was $44.11 \%$ $(15 / 34)$ for Tc99 tracer and $57.1 \%$ for ICG(16/28).

Overall rate of lymph node involvement was 10,6\% (5/47). Macroscopic lymph node pelvic metastasis were found in 4 patients $(8.5 \%)$ and in only 1 case $(2.1 \%)$ microdisease in SLN was found. A $30.7 \%$ (4/13) who para-aortic SLN was not detected, had infiltration of para-aortic lymphadenectomy. Two of them had also pelvic SLNs infiltration. There was a $4.2 \%$ positive para-aortic lymphadenectomies with negative pelvic lymph nodes.

Conclusion* The SLNs biopsy with dual tracer and dual injection (cervix and fundus) offers good overall detection and could increase para-aortic detection compared with cervical injection alone. When SLNs is not detected in para-aortic area we should complet the lymphadenectomy because in our study the rate of positive lymphadenectomy is not negligible (30\%). A dual tracer could help in the ICG learning curve and ensure that the right SLN is removed.

\section{DIAGNOSTIC ACCURACY OF INTRAOPERATIVE HISTOPATHOLOGICAL ASSESSMENT IN ENDOMETRIAL CANCER}

${ }^{1} \mathrm{M}$ Plancha, ${ }^{1} \mathrm{~A}$ Miguel, ${ }^{1 ; 2} \mathrm{D}$ Djokovic, ${ }^{3} \mathrm{PA}$ Duarte, ${ }^{3} \mathrm{C}$ Barros, ${ }^{3 ; 4} \mathrm{P}$ Pinto* ${ }^{*}$ Alfredo da Costa Maternity Hospital, Gynecology, Lisboa, Portugal; ${ }^{2}$ Nova Medical School, Ob/Gyn, Lisboa, Portugal; ${ }^{3}$ Alfredo da Costa Maternity Hospital, Lisboa, Portugal; ${ }^{4}$ First Faculty of Medicine Charles University, Prague, Czech Republic

\subsection{6/ijgc-2021-ESG0.227}

Introduction/Background* Endometrial cancer (EC) is the most common gynecologic malignancy in developed countries and the main treatment consists of surgery. Myometrial invasion is commonly used in guiding surgical extent and can be evaluated by intraoperative assessment (IOA). There are studies that show different reports regarding its adequacy and reliability. The aim of this study was to assess the diagnostic accuracy of IOA comparing with definitive histopathologic report surgically resected EC.

Methodology We analysed retrospectively all patients submitted to surgical staging for presumed EC with a definitive diagnosis of endometrioid carcinoma grade 1 and 2 at a tertiary hospital in 2017-2020. Results of IOA were compared to the final pathology report. The accuracy, sensitivity, specificity, positive predictive value (PPV) and negative predictive value (NPV) of the IOA were calculated.

Result(s)* A total of 97 patients underwent surgical staging for EC. We had 41 women with histologically confirmed endometrioid carcinoma in which 39 were performed the IOA. The IOA had an accuracy of $82,1 \%$, sensitivity of $64,3 \%$, specificity of $92 \%$, PPV of $81,8 \%$ and NPV of $82,1 \%$ in predicting miometrial invasion. In the definitive histopathological report 26 patients were classified with stage IA and 13 with stage IB. In the 27 cases identified as stage IA in IOA, the pathology reported stage IB in 4/39 (10,2\%). 11/39 (28,2\%) cases were identified as stage IB in IOA, and only $2 / 39(5,1 \%)$ were in fact stage IA in the final pathology report. Surgery, based on the result of the IOA, caused undertreatment in 4/ $39(10,2 \%)$ and overtreatment in $2 / 39(5,1 \%)$ patients.

Conclusion* Our IOA results have reasonable accuracy rates in predicting malignancy. However, the concordance of tumor myometrial invasion between IOA and pathology report was suboptimal, leading to the need of a secondary surgery in some patients.

\section{ABSTRACT WITHDRAWN}

\section{CARDIAC METASTASIS OF ENDOMETRIAL CANCER: A MULTIDISCIPLINARY APPROACH}

${ }^{1}$ C Cipullo*, ${ }^{2} \mathrm{P}$ Campisi, ${ }^{3} \mathrm{P}$ Centofanti, ${ }^{4} \mathrm{G}$ Musumeci, ${ }^{5} \mathrm{~S}$ Cirillo, ${ }^{1} \mathrm{~J} O \mathrm{~S}$ Pezua Sanjinez, ${ }^{1} \mathrm{~N}$ Biglia, ${ }^{1} \mathrm{~A}$ Ferrero. 'Mauriziano Umberto Hospital, Academic Department Gynaecology and Obstetrics, Torino, Italy; ${ }^{2}$ Mauriziano Umberto Hospital, Division of Pathology, Torino, Italy; ${ }^{3}$ Mauriziano Umberto Hospital, Division of Cardiac Surgery, Torino, Italy; ${ }^{4}$ Mauriziano Umberto Hospital, Division of Cardiology, Torino, Italy; ${ }^{5}$ Mauriziano Umberto Hospital, Department of Radiology, Torino, Italy

\subsection{6/ijgc-2021-ESG0.228}

Introduction/Background* Literature on cardiac metastases from endometrial cancer (EC) is scarce. We report a unique case of a 65-years-old woman diagnosed endometrioid EC, G3, FIGO stage IIIC, treated with surgery and adjuvant chemo-radiation in 2018. In April 2020, while she was undergoing chemotherapy (CHT) with Carboplatin-Paclitaxel because of abdominal and oropharyngeal recurrence, a F18 FDG-PET/ CT showed a partial response of the known lesions, but highlighted high glucose uptake in the right atrium, deserving timely diagnostic investigation.

Methodology A multidisciplinary discussion was undertaken to choose the best management: gynecologists, cardiologists,

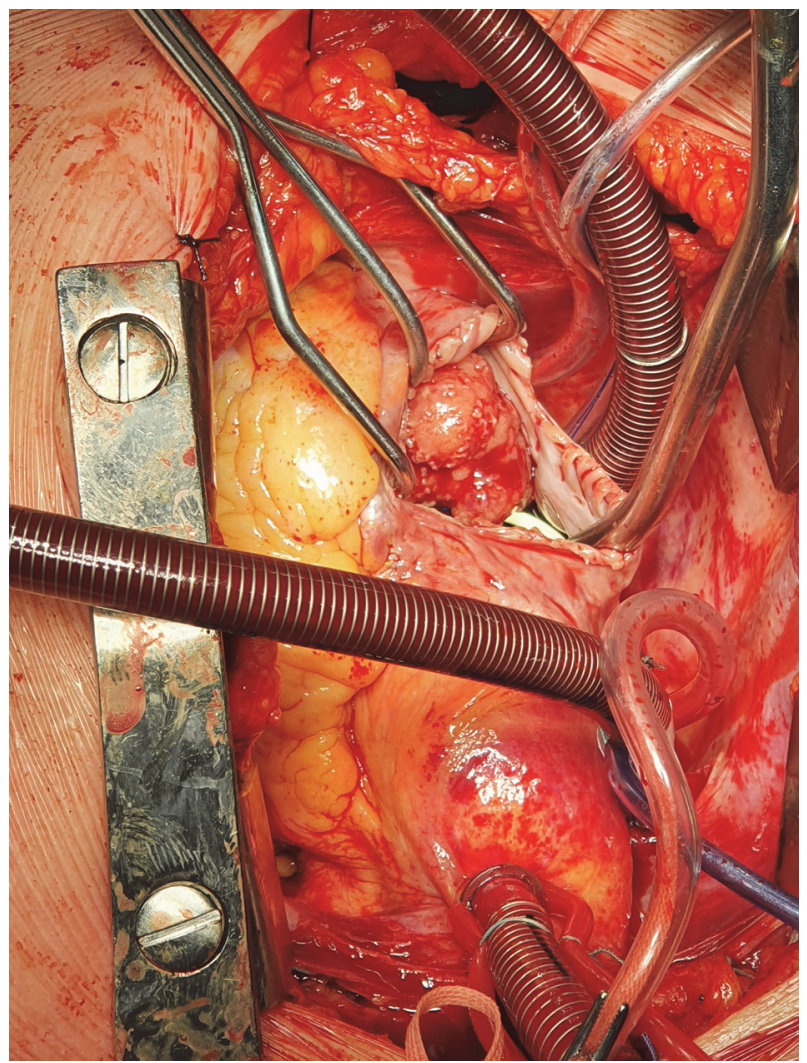

Abstract 1089 Figure 1 


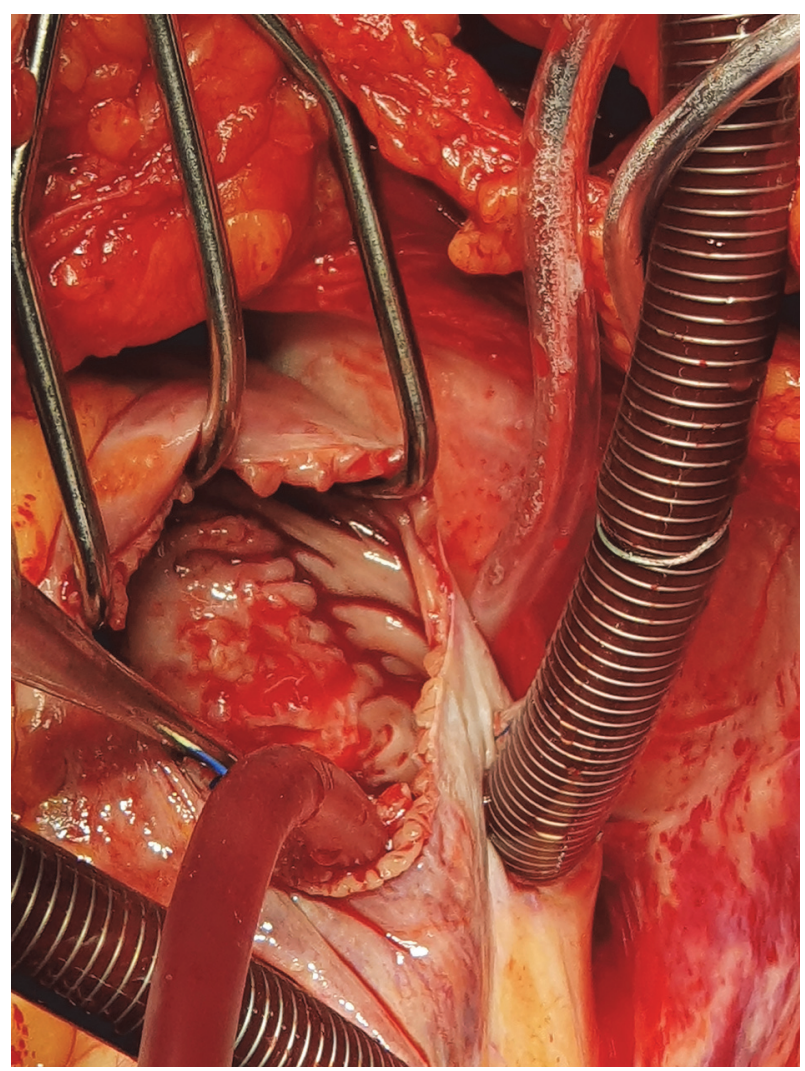

Abstract 1089 Figure 2

cardiac surgeons, pathologists, oncologists and cardiovascular anesthesiologists were involved. A complete trans-thoracic echocardiogram was performed, showing an isoechoic, mobile, adherent to the tricuspid ring, 22 x $50 \mathrm{~mm}$ mass with nonsmooth margins, resulting in obstruction of the right ventricular filling (gradient $7 \mathrm{mmHg}$ ). Cardiac magnetic resonance imaging (MRI) demonstrated a 41x35 mm, polylobed mass, adhered to the tricuspid ring on the inferior-posterior side, which incorporated the posterior and septal flaps of the tricuspid valve, extensively emerging in the ventricular cavity in the diastolic phase. The mass obstructed the right ventricular filling and its most caudal portion partially occupied, in the systolic phase, the outlet of the inferior vena cava in the atrium. Because of the life-threatening obstruction, the mass was removed by cardiac surgeons with sternotomy in extracorporeal circulation. During surgery, the mass was analyzed by pathologist, confirming the endometrial origin. Postoperatively, radiotherapy (RT) on the heart was performed, followed by second line CHT with Liposomal Doxorubicin till February 2021.

Result(s)* In February 2021 cardiac MRI was performed, showing no evidence of disease. Complete response of the oropharyngeal and abdominal recurrences was also detected. But the woman presented with neurological symptoms, as partial paralysis of the legs. Encephalic MRI was performed showing brain metastases. The woman underwent pan-encephalic RT with subsequent reduction of those lesions.

In June 2021, the woman is still alive without neurological nor other symptoms.

Conclusion* A multidisciplinary approach is essential to treat rare EC metastases. Reference centers for the treatment of EC must have specialists from other disciplines available, to ensure the best clinical practice.

\section{OPTIMISING OUTCOMES FOR LAPAROSCOPIC HYSTERECTOMY IN PATIENTS WITH MORBID OBESITY}

E Van der Zanden, F Testa, C White, P Larsen-Disney, F Drews, S Kaushik, S Baron* University Hospitals Sussex NHS Foundation Trust, Gynaecological Oncology, UK

\subsection{6/ijgc-2021-ESG0.229}

Introduction/Background* Operating on patients with a significantly raised body mass index (BMI) represents a significant challenge to the surgical and the anaesthetic team. Hysterectomy for early-stage uterine cancer is usually performed via laparoscopy.

We aimed to evaluate whether a two consultant 'buddy operating' approach improves on intra-operative and postoperative outcomes in patients undergoing total laparoscopic hysterectomy (TLH) for endometrial cancer who are morbidly obese.

Methodology A prospectively selected cohort of 25 patients with a BMI 47-70 undergoing TLH was divided into two groups according to whether the first assistant to the GynaeOncology consultant was a registrar (ST3-7), or a consultant ('buddy operating'). Anaesthetic time, operating time, intraoperative estimated blood loss (EBL), requirement for high dependency unit (HDU) bed and length of stay (LOS) were compared in the two groups.

Result(s)* Average 'buddy' operating time was significantly shorter compared to the registrar-assistant group $(01: 31 \mathrm{~h}$ vs $01: 59$ h respectively; $\mathrm{p}<0.001)$; a similar trend was seen with the average total anaesthetic time $(02: 48 \mathrm{~h}$ vs $03: 23 \mathrm{~h}$ respectively; $\mathrm{p}<0.001)$. EBL was less in the 'buddy operating' group $(39 \mathrm{mls})$ vs registrar-assistant group $(169 \mathrm{mls}$; $\mathrm{p}<0.001)$. Postoperatively, LOS was shorter in the 'buddy operating' group as compared to the registrar-assistant, though not significantly so $(1.13$ vs 1.59 days; $p=0.109) .2$ of the total patients $(8 \%)$ required a one-night stay in HDU for observation due to their co-morbidities, both in the registrar-assistant group. Mean BMI, age, ASA and comorbidities were similar in the two groups.

Conclusion* In patients with a significantly raised BMI, TLHs by two consultants vs consultant and registrar are associated with better intra and post-operative outcomes, including reduced overall anaesthetic time, operating time, and EBL. There is an association with a reduced length of overall hospital stay, though this was not significant.

\section{IMPLICATIONS OF OBESITY IN ENDOMETRIAL CANCER}

${ }^{1 ; 2} \mathrm{MC}$ Tirnovanu*, ${ }^{3 ; 4} \mathrm{D}$ Timofte, ${ }^{5} \mathrm{R}$ Corduneanu, ${ }^{5} \mathrm{~A}$ Crudu, ${ }^{5} \mathrm{~B}$ Scurtu, ${ }^{6} \mathrm{~A}$ lov, ${ }^{7} V G$ Tîrnovanu. 'University of Medicine and Pharmacy "Gr T Popa', Obstetrics and Gynecology, lasi, Romania; '2Clinical Hospital of Obstetrics and Gynecology „,Cuza Vodă', Iasi, Romania; 'University of Medicine and Pharmacy "Gr T Popa', Surgery, lasi, Romania; ${ }^{4}$ Clinical Hospital "Sfântul Spiridon' laşi, Surgery, lasi, Romania; ${ }^{5}$ Clinical Hospital of Obstetrics and Gynecology "Cuza Vodă", Obstetrics and Gynecology, lasi, Romania; ${ }^{6}$ University of Medicine and Pharmacy "Gr T Popa', student sixth year, lasi, Romania; 'University of Medicine and Pharmacy ",Gr T Popa', student fourth year, lasi, Romania

\subsection{6/ijgc-2021-ESG0.230}

Introduction/Background* Over half of the endometrial cancers (EC) are caused by obesity, known as an independent risk factor. In Romania, in the last years the obesity rate had an alarming growth, with many high grade cases that influence the clinical management of the disease, the surgical procedure, radiotherapy planning, and the survival rate. 